\title{
Anomalous Relaxation in Polychlorinated Biphenyl/Polybutadiene Mixtures
}

\author{
P. G. Santangelo, K. L. Ngai, and C. M. Roland* \\ Naval Research Laboratory, Washington, D.C. 20375-5342 \\ Received November 15, 1993; Revised Manuscript Received February 24, $1994^{\circ}$
}

\begin{abstract}
The phenomenon of faster relaxation of small-molecule glass-forming liquids upon addition of a higher $T_{\mathrm{a}}$ polymer is examined for mixtures of Aroclor 1248 (PCB) with poly(vinylethylene) (PVE). In this system the anomaly can be ascribed to the positive changes in volume on mixing. Contributions to the observed behavior from changes in local friction coefficient, as deduced from the coupling model of relaxation, exert a less obvious influence. This can be contrasted with prior results on mixtures of poly(methylphenylsiloxane) (PMPS) with the small-molecule glass former 1,1-bis( $p$-methoxyphenyl)cyclohexane (BMC). ${ }^{1,2}$ Despite a similarly anomalous solvent modification, the volume change upon mixing is negative therein. This suggests that changes in local friction, as inferred from comparison of the components' noncooperative relaxation times, are a general mechanism contributing to the local dynamics in mixtures.
\end{abstract}

\section{Introduction}

The longstanding view that dilute polymer concentrations do not affect solvent rotational mobility has yielded to recent experimental evidence. The presence of polymer can significantly modify the local friction coefficient and solvent rotational dynamics. ${ }^{3-7}$ An unusual form of this modification was recently seen ${ }^{8}$ in polychlorinated biphenyl (tradenamed Aroclor) containing a low concentration of dissolved 1,2-polybutadiene. The common expectation is that a solvent-rich mixture containing a higher $T_{\mathrm{g}}$ polymer will exhibit an increased relaxation time over that of the neat solvent; that is, if the local polymer dynamics are slower than the diluent dynamics, the latter can be slowed down by addition of the polymer. This behavior is invariably observed for mixtures of liquids having very different glass transition temperatures. The glass transition temperature of the mixture, as well as the relaxation times of all components, falls between those of the neat liquids. Dynamic depolarized light scattering measurements ${ }^{8}$ established for a solvent-rich $\mathrm{PCB} / \mathrm{PVE}$ mixture that the polymer not only alters the dynamic properties of the small-molecule solvent but unexpectedly decreases the PCB reorientational relaxation time. The observed enhancement in the solvent mobility is remarkable in that the $T_{\mathrm{g}}$ of the PVE was approximately $19 \mathrm{deg}$ above that of neat PCB.

An interpretation of this phenomenon was presented 8 based on the coupling model of relaxation. 9,10 According to the coupling model, the observed relaxation time, $\tau^{*}$, depends on both the local friction and the extent to which nonbonded neighboring molecules constrain the local motion

$$
\tau^{*}=\left[(1-\mathrm{n}) \omega_{\mathrm{c}}{ }^{\mathrm{n}} \tau_{0}\right]^{1 /(1-\mathrm{n})}
$$

where $\omega_{c}{ }^{-1}$ defines the inception of intermolecular constraints and $n$ is a measure of the severity of intermolecular constraints. The local friction factor is reflected in the magnitude of $\tau_{0}$, the relaxation time in the absence of intermolecular constraints. Recent quasielastic neutron scattering experiments performed on poly(vinyl chloride) ${ }^{11}$ provide direct evidence of a slowing down in the local dynamics at a temperature insensitive time, $\omega_{c}{ }^{-1}$.

\footnotetext{
- Abstract published in Advance ACS Abstracts, June 1, 1994.
}

Table 1. Polymer Samples

\begin{tabular}{lrcccc}
\hline polymer & mol wt & \% vinyl groups & $T_{\mathrm{g}}{ }^{b}(\mathrm{~K})$ & $T_{\mathrm{g}}{ }^{c}(\mathrm{~K})$ & $n^{d}$ \\
\hline PVE-1 & 27600 & 93 & 268 & 261.3 & 0.64 \\
PVE-h & 153000 & 96 & 274 & 264.4 & 0.65 \\
PBD-h & 387000 & 83 & 258 & 250.3 & 0.63 \\
PCB & & & 239 & 228.6 & 0.41
\end{tabular}

${ }^{\circ}$ The remaining chain units are 1,4-polybutadiene. ${ }^{b}$ Differential scanning calorimetry at $10 \mathrm{~K} / \mathrm{min}$. ${ }^{c}$ The temperature at which the mechanical relaxation time equals $100 \mathrm{~s}$. $d$ These values are consistent with previously reported results. ${ }^{12,13}$

Since a distribution of local environments exists in a mixture, the observed relaxation time reflects an average; that is, $\tau^{*}$ is the relaxation time for the nominal composition. According to the coupling model, PVE segmental relaxation is highly cooperative (ascribed to the presence of the sterically hindering pendant vinyl groups ${ }^{12,13}$ ), in contrast to $\mathrm{PCB}$, which is not as strongly intermolecularly coupled (smaller $n$ ). While chlorinated biphenyls are regarded as a high-viscosity fluids and even associated with clustering, ${ }^{14-16}$ in the framework of the coupling model, such aspects are subsumed in the "static mean field" part of the intermolecular interaction. In combination with any intramolecular energy barriers, this determines the value of $\tau_{0}$ in eq 1 and should be distinguished from the constraint dynamics part underlying the magnitude of the coupling parameter $n$.

Even though the PVE has a higher $T_{\mathrm{g}}$ than the PCB, the nonlinearity of eq 1 suggests that this is due to its larger coupling parameter; the polymer may have a smaller friction coefficient (i.e., smaller $\tau_{0}$ ). The strong intermolecular coupling of the neat polymer is alleviated by dilution, whereby its relaxation time could actually become shorter than that of the PCB. This was demonstrated ${ }^{8}$ using eq 1 , where the relaxation time in the absence of intermolecular constraints was predicted to be less for PVE than for PCB, $\tau_{0}(\mathrm{PVE})<\tau_{0}(\mathrm{PCB})$. Hence, the coupling model predicts a reversal in relaxation times since $\tau^{*}(\mathrm{PVE})>\tau^{*}(\mathrm{PCB})$. This implies that addition of the higher $T_{\mathrm{g}}$ polymer would speed up relaxation of the PCB. In their application of the coupling model, Rizos and $\mathrm{Ngai}^{8}$ gave no consideration to possible modification of $\tau_{0}$ 's due to the nonzero excess volume of mixing (i.e., a nonideal mixing volume $e^{7,17,18}$ ).

Recently, similar coupling model-derived arguments were advanced to explain the anomalous solvent modification in mixtures of poly (methylphenylsiloxane) (PMPS) with a small-molecule glass former, 1,1-bis( $p$-methoxy- 


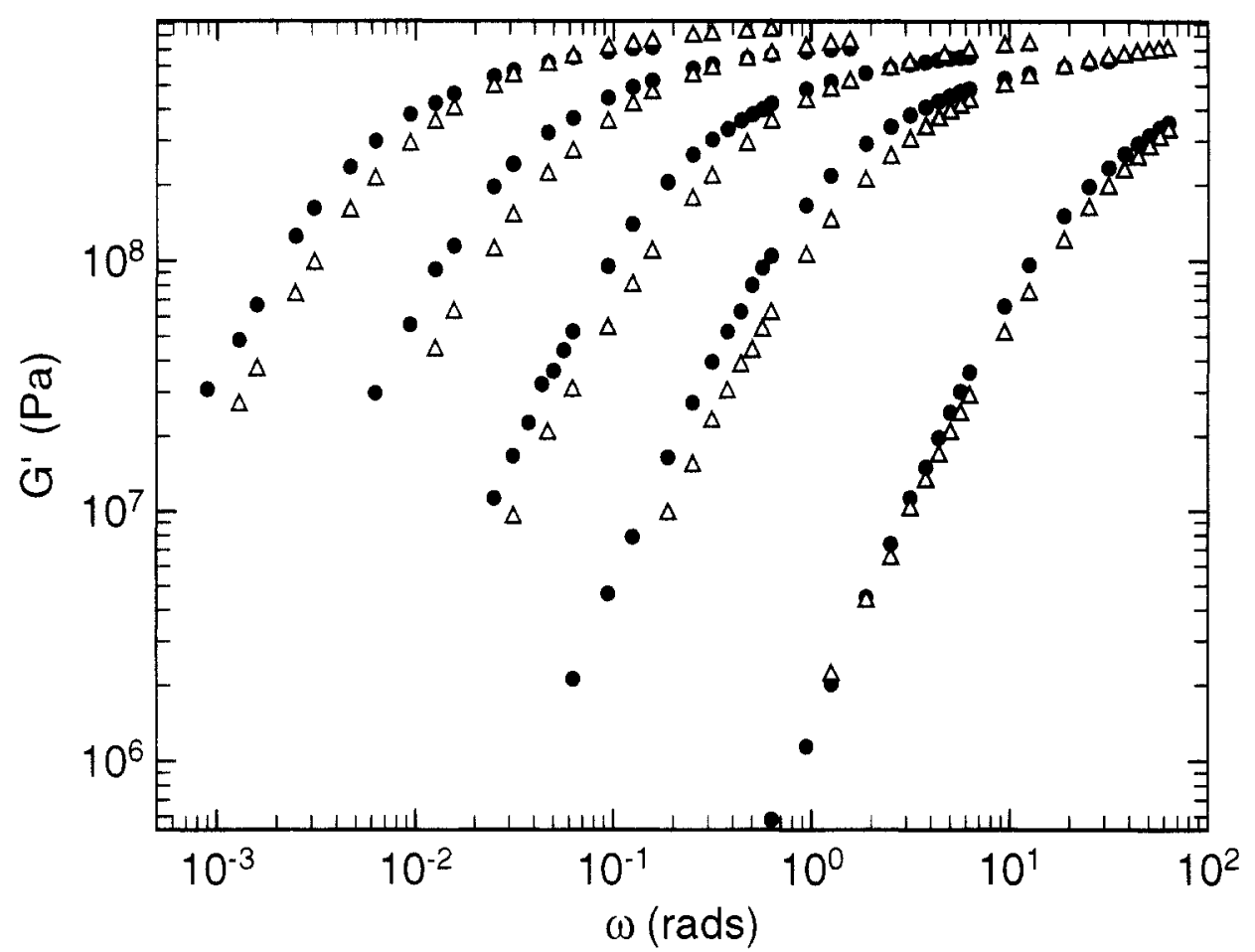

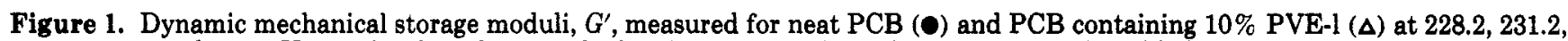
$234.2,237.2$, and $242.7 \mathrm{~K}$ (moving from lower to higher frequency). At each temperature, the added polymer causes a slight shift to higher frequency.

phenyl)cyclohexane (BMC). ${ }^{2}$ The reorientational dynamics of BMC are faster in the presence of higher $T_{\mathrm{g}}$ PMPS. While nonzero excess volume can modify the solution dynamics, ${ }^{7,20}$ BMC densifies when mixed with a small concentration of PMPS. ${ }^{2}$ On the basis of free volume ideas, $7,21,22$ this negative excess volume should slow down the BMC motion, contrary to the experimental results. In the case of BMC/PMPS, the coupling model offers the only tenable hypothesis for the observed speeding up of the BMC relaxation.

We have extended the work on the PVE/PCB system, changing both the vinyl content of the PVE and its molecular weight from that used in ref 8 . In this fashion, the coupling parameter $n$ and the noncooperative relaxation time, $\tau_{0}$, could be systematically varied. This enables us to probe the origin of the anomalous speeding up of $\mathrm{PCB}$ reorientation in the presence of the higher $T_{\mathrm{g}}$ polymer.

\section{Experimental Section}

The polybutadienes used were obtained from the Firestone Tire and Rubber Co. The vinyl content of these atactic, random copolymers are listed, along with their weight-average molecular weights and glass transition temperatures, in Table 1 . We refer to the highest vinyl polybutadienes as PVE (poly(vinylethylene)), while the polymer having more (ca. 17\%) 1,4-microstructure is referred to as PBD. Higher and lower molecular weights are designated by - $h$ and -1 , respectively (note that all polymers used herein had higher molecular weights, and thus higher $T_{\mathrm{g}}{ }^{\prime} \mathrm{s}$, than the PVE in ref 8). The PCB was Aroclor 1248 from the Monsanto Chemical Co., a glass-forming liquid consisting of randomly chlorinated biphenyls (average chlorine content $=48 \%$ ). Mixtures were prepared by dropwise addition of the PCB onto cast films of the polybutadiene, followed by annealing with periodic mechanical agitation for several weeks at room temperature.

Dynamic mechanical data in the vicinity of the glass transition zone were obtained with a Bohlin VOR rheometer using a parallelplate geometry. Sample radii and gaps were typically 6 and 2 $\mathrm{mm}$, respectively. Prior to measurements at any given temperature, samples were maintained for a time period exceeding the anticipated relaxation time. Reproducibility was affirmed by repeat measurements after varying time periods.
Room temperature specific gravities, relative to propanol, were obtained using the buoyancy method (ASTM method D792 A-1) and by pycnometry (ASTM designation D792 A-2). Sample masses exceeding $0.1 \mathrm{~g}$ were used in conjunction with a Mettler AE163 balance (accuracy $=0.1 \mathrm{mg}$ ), yielding four significant figures for the density determinations.

\section{Results and Discussion}

The dynamic mechanical storage and loss moduli, measured at various temperatures, are plotted in Figures 1 and 2 for both neat PCB and a mixture containing $10 \%$ PVE-l. Notwithstanding the higher $T_{\mathrm{g}}$ of PVE-1, the PCB reorientational relaxation time is observed to decrease upon mixing. Also shown in the semilogarithmic plots of Figure 2 are the best fits to the Kohlrausch-WilliamsWatts stretched exponential function ${ }^{23,24}$

$$
E(t)=\exp \left[-\left(\frac{\mathrm{t}}{\tau^{*}}\right)^{1-\mathrm{n}}\right]
$$

using

$$
E^{\prime \prime}(\omega)=\omega \int_{0}^{\infty} E(t) \cos (\omega t) \mathrm{d} t
$$

In none of these measurements was there any evidence of multiple glass transitions, as has been reported for a few other mixtures of small molecules with polymers. ${ }^{16,25-29}$ This is illustrated in Figure 3, which shows the dynamic moduli of the $50 / 50$ mixture measured at a fixed frequency over a wide range of temperatures. Only one transition is observed. Note that the $\tau^{*}$ in eqs 1 and 2 is very nearly equal to $\left(2 \pi f_{\mathrm{p}}\right)^{-1}$, where $f_{\mathrm{p}}$ is the frequency of the maximum in the dynamic loss modulus.

According to the coupling model, $, 9,10$ broader dispersions (larger $n$ values) for amorphous polymers are associated with more intermolecular cooperativity, while a narrow dispersion implies weaker constraints on the relaxation from nonbonded neighbors. The best-fit values for $n$ are listed in Table 1 . The magnitude of $n$ was only weakly dependent on temperature, varying no more than \pm 0.02 


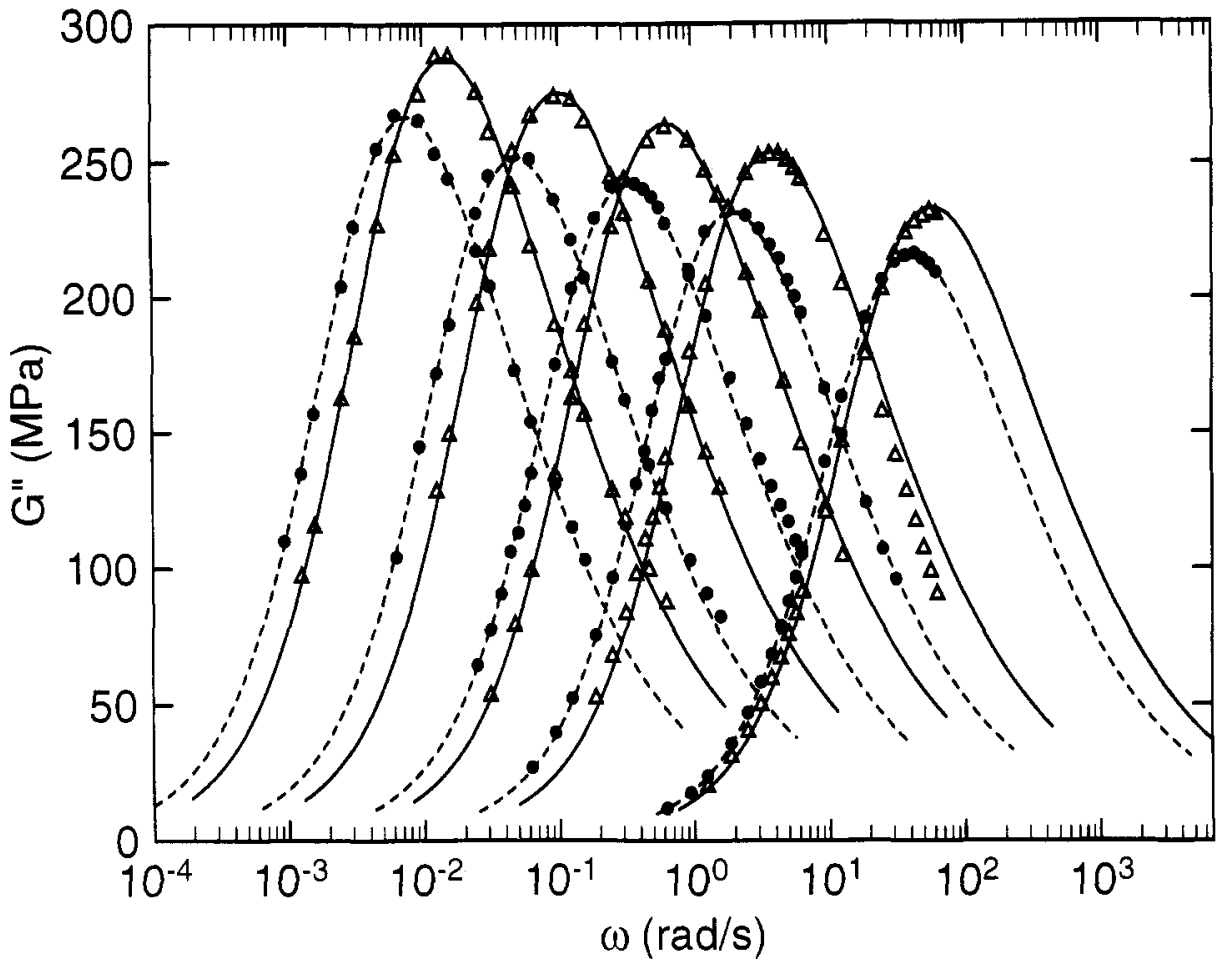

Figure 2. Dynamic mechanical loss moduli, $G^{\prime \prime}$, measured for neat PCB $(\bullet)$ and PCB containing $10 \%$ PVE-l $(\Delta)$ along with the fit to eq 2. The data correspond to the temperatures given in Figure 1. At each temperature, the added polymer causes a slight increase in amplitude and a shift to higher frequency. Even though the added polymer has a higher $T_{\mathrm{g}}$, the PCB relaxation time decreases for the mixture.

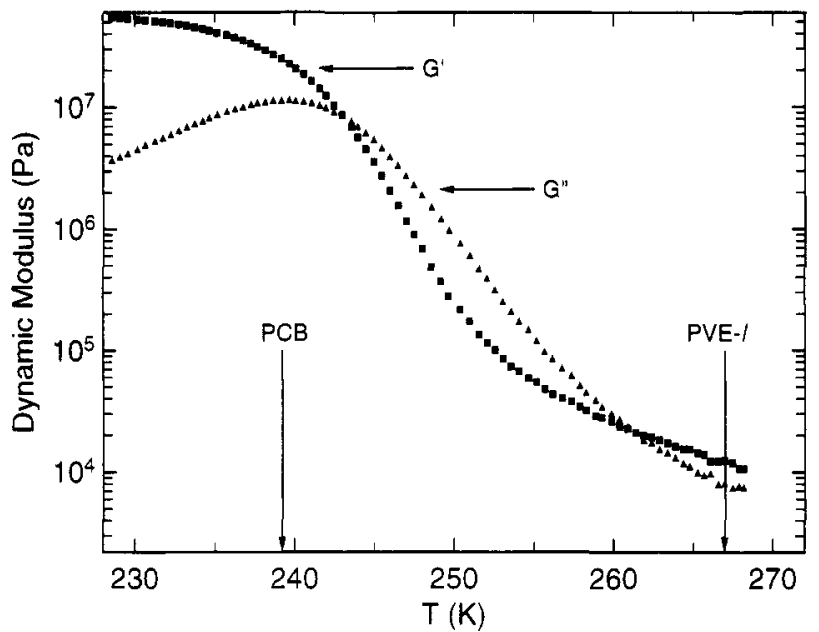

Figure 3. Temperature dependence of the dynamic mechanical moduli measured at $1 \mathrm{~Hz}$ for PCB containing $50 \%$ PVE-l. Only one transition is observed for the mixture. The glass transition temperatures of the neat components are indicated by the vertical arrows.

over the range of measurement temperatures. The results for the neat polymers are consistent with previously reported values. ${ }^{12,13}$

Displayed in Figure 4 is the temperature dependence of the relaxation times measured for various compositions of PCB with the low molecular weight PVE-1. Also included in Figure 4 are the best fits of the Vogel-Fulcher equation $^{21}$

$$
\tau^{*}=\mathrm{A} \exp \left(\frac{B}{T-T_{\infty}}\right)
$$

Figure 4 has two interesting features. First, the PCB-rich mixtures ( $>75 \% \mathrm{PCB}$ ) possess shorter relaxation times than neat $\mathrm{PCB}$; that is, addition of a higher glass transition temperature component decreases the relaxation time of the PCB. Second, the $50 \%$ composition exhibits a relaxation time near that of neat PCB; the composition dependence of the relaxation times is highly skewed. Using an operational definition of $T_{\mathrm{g}}$ as the temperature at which the relaxation time equals $100 \mathrm{~s}$ (with eq 4 used to interpolate the experimental data), the glass transition temperatures of the PCB-rich mixtures $(>75 \%$ PCB) are found to be less than $T_{\mathrm{g}}$ of neat PCB (Figure 5). This is contrary to the expectation that mixture transition temperatures will be intermediate to those of the pure components.

The anomalous relaxation behavior seen in Figures 1 , 2,4 , and 5 is analogous to results obtained on the PMPS/ $\mathrm{BMC}^{1,2}$ and low molecular weight PVE/PCB systems ${ }^{8}$ discussed earlier, where the addition of a small quantity of higher $T_{\mathrm{g}}$ polymer also shortens the relaxation time. It was proposed therein that the reversal predicted from eq 1 in going from $\tau^{*}$, the relaxation time observed for the neat material, to the noncooperative relaxation time $\tau_{0}$ underlies the anomaly. The idea is that while $\tau^{*}$ depends both on the strength of the intermolecular coupling (i.e., $n$ ) and on the local friction coefficient (as reflected in $\tau_{0}$ ), alleviation of the intermolecular constraints in the neat polymer upon mixing makes irrelevant the value of $\tau^{*}$ (and $T_{\mathrm{g}}$ ) measured for the neat polymer. This implies that the nature of the solvent modification must be gauged from the relative magnitudes of the component $\tau_{0}$ 's.

Considering PVE-l and PVE-h, it has been previously demonstrated $\mathrm{d}^{30,31}$ that polymers having the same chemical structure will exhibit equivalent coupling parameters; however, the individual $\tau^{*}$ 's (and hence the glass transition temperatures) are not the same if the molecular weights, and $\tau_{0}$ 's, are different (Table 1). As a result, the reversal reported for a very low molecular weight $\mathrm{PVE}$ with $\mathrm{PCB},{ }^{8}$ whereby at the same temperature

$$
\tau^{*}(\mathrm{PVE}) \gg \tau^{*}(\mathrm{PCB})
$$

but 


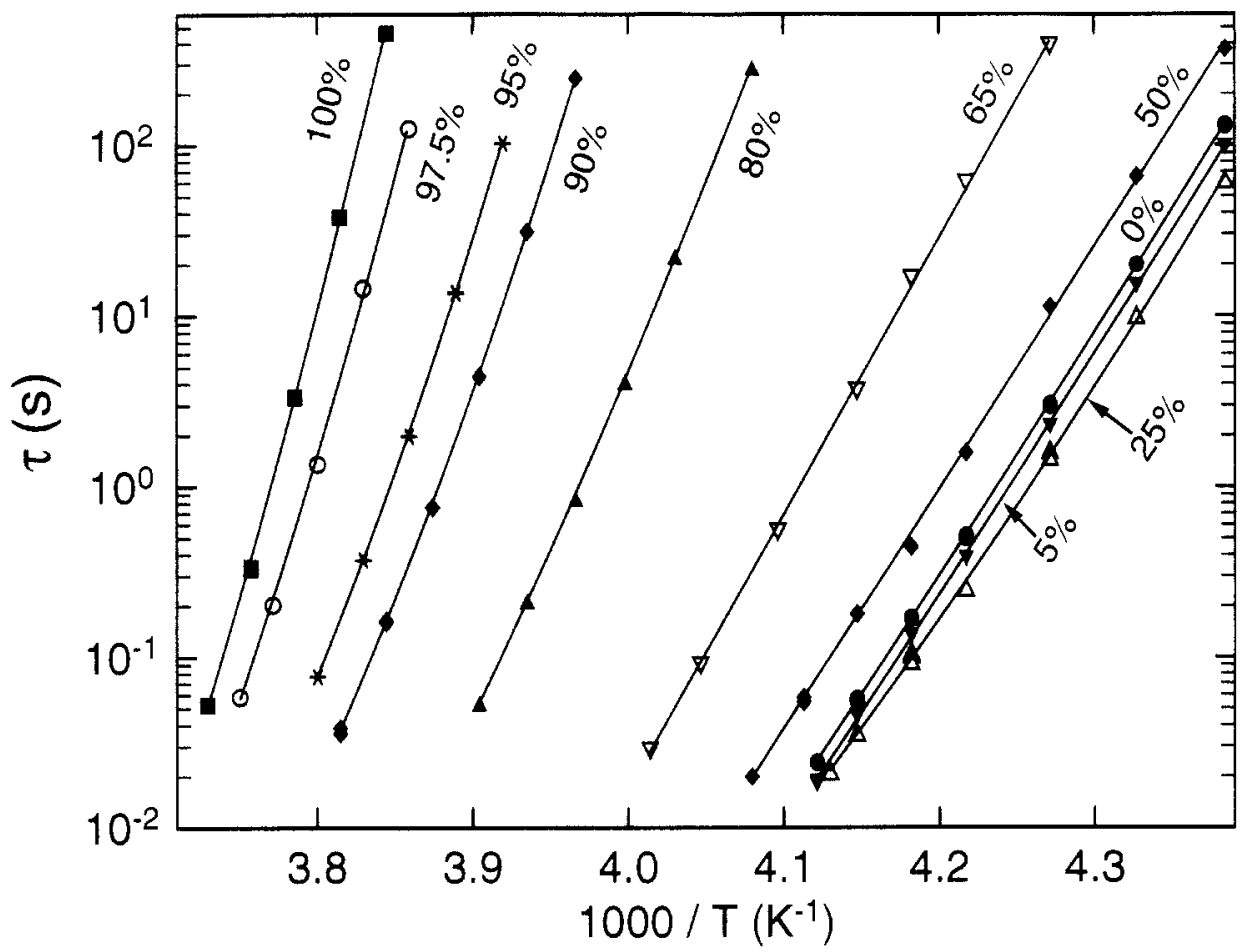

Figure 4. Relaxation times for PCB, PVE-1, and their mixtures as a function of inverse temperature. The concentration by weight of polymer is indicated, with the solid curves representing the fit to eq 4. Anomalous relaxation behavior is seen for all PCB-rich mixtures.

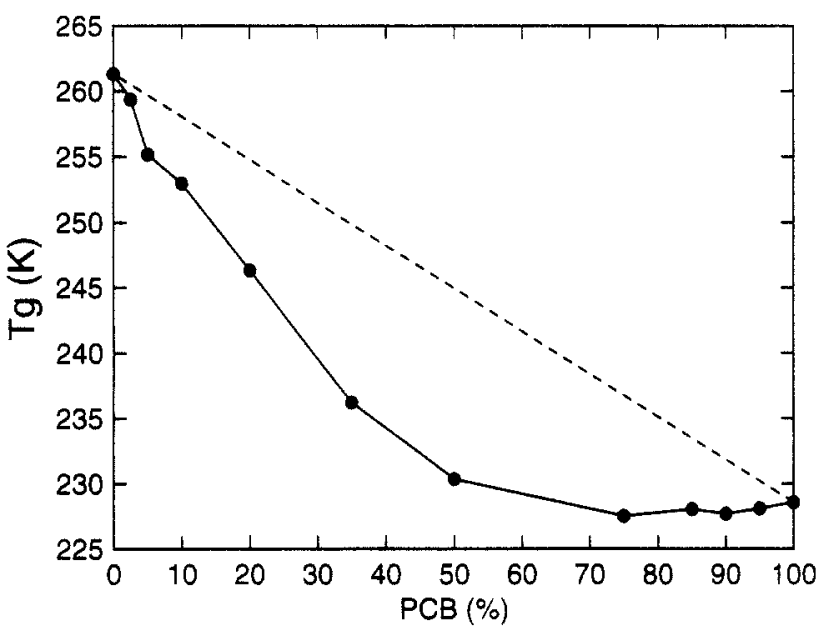

Figure 5. Composition dependence (by weight) of the glass transition temperatures, taken as the temperature at which the relaxation time equals $100 \mathrm{~s}$, with eq 4 used to interpolate the measured data. Note that the $\mathrm{T}_{\mathrm{p}}$ 's of the PCB-rich mixtures $(>75 \% \mathrm{PCB})$ are less than neat PCB.

$$
\tau_{0}(\mathrm{PVE}) \ll \tau_{0}(\mathrm{PCB})
$$

is calculated (eq 1) to not inhere in either of the present cases (PVE-h or PVE-l). That is,

$$
\begin{aligned}
& \tau^{*}(\mathrm{PVE}-\mathrm{h}) \gg \tau^{*}(\mathrm{PCB}) \\
& \tau^{*}(\mathrm{PVE}-\mathrm{l}) \gg \tau^{*}(\mathrm{PCB})
\end{aligned}
$$

and

$$
\begin{aligned}
& \tau_{0}(\mathrm{PVE}-\mathrm{h})>\tau_{0}(\mathrm{PCB}) \\
& \tau_{0}(\mathrm{PVE}-\mathrm{l})>\tau_{0}(\mathrm{PCB})
\end{aligned}
$$

There is no reversal in the magnitudes of the cooperative $\left(\tau^{*}\right)$ and noncooperative $\left(\tau_{0}\right)$ relaxation times, although the mismatch in the time scales of PVE and PCB motions is still significantly reduced from the degree expected from simple $T_{\mathrm{g}}$ (or $\tau^{*}$ ) considerations. Nevertheless, the prediction is that addition of the polymer should slow down the PCB relaxation; notwithstanding, Figure 4 reveals that anomalous speeding up of the $\mathrm{PCB}$ reorientation occurs upon addition of the PVE. This indicates that some other mechanism also contributes to the anomalous behavior seen herein.

We also examined the effect on the PCB dynamics of addition of a polybutadiene having lower 1,2-content. Due to its lower concentration of pendant vinyl groups, this PBD-h (Table 1) has a lower $T_{\mathrm{g}}$ and smaller $n .^{30}$ This means that its $\tau^{*}$ will be closer to that of PCB; however, given the nonlinear dependence on $n$ (eq 1), still $\tau_{0}(\mathrm{PBD}$ h) $>\tau_{0}(\mathrm{PCB})$. Nevertheless, the anomaly of faster PCB relaxation upon addition of higher $T_{\mathrm{g}}$ polymer persists (Figure 6).

The mechanism proposed previously, ${ }^{8}$ that the noncooperative relaxation time of the polymer is smaller than $\tau_{0}(\mathrm{PCB})$, cannot in this simple form explain the present results. A nonideal volume change of mixing (i.e., positive excess volume $\mathrm{e}^{17-19}$ ) is potentially the other factor governing the changes in PCB relaxation times. To assess this influence herein, the densities of the pure PCB and PVE. 1 , along with 50 and $80 \%$ PCB-rich mixtures, were measured. The results (Figure 7) reveal that the density of the PCB decreases on addition of polymer to a value less than the arithmetic mean of the pure component densities. While densification (mixture densities greater than the linearly interpolated value from the pure components) is more commonly observed for mixtures of small molecules with polymers, ${ }^{17-19}$ positive volume changes have on occasion been reported for polymer solutions. ${ }^{32}$ From a simple free volume interpretation, $7,21,22$ a positive excess volume should increase the mobility of the PCB, in accord with the experimental results.

\section{Summary}

We conclude that a positive volume change of mixing is primarily the cause of the faster PCB relaxation when 


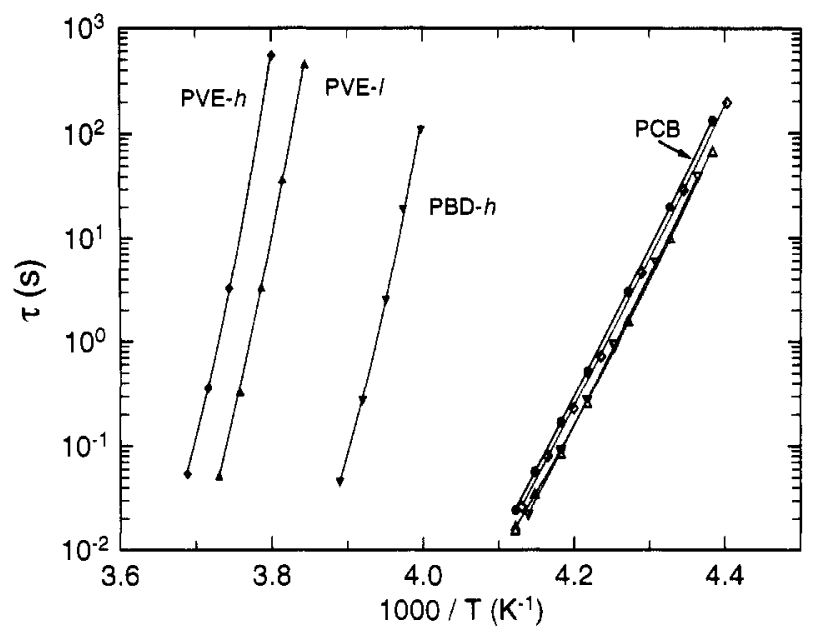

Figure 6. Segmental relaxation times for the $\operatorname{PCB}(\bullet)$, the neat polybutadienes (solid symbols), and PCB containing $10 \%$ polymer (corresponding hollow symbols). All mixtures exhibit the anomalous speeding up of $\mathrm{PCB}$ reorientation upon addition of higher $T_{\mathrm{g}}$ polymer.

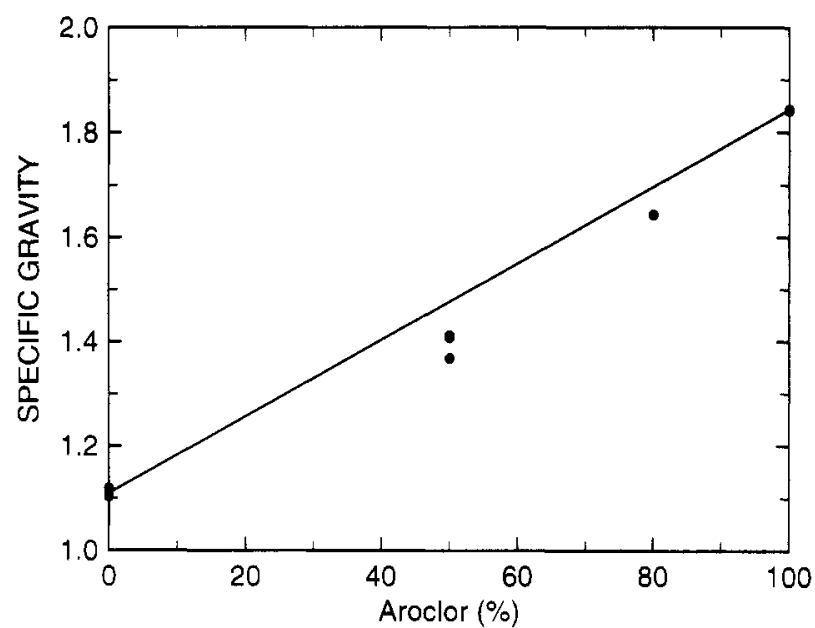

Figure 7. Density of neat PVE-1, neat PCB, and 50 and $80 \%$ PCB-rich mixtures at room temperature. Three measurements at each composition are illustrated, although the results closely overlap. The solid line represents linear interpolation of the pure component densities. Note that addition of PVE-1 is associated with a positive excess volume, consistent with the observed decrease of PCB's relaxation time.

mixed with high-vinyl polybutadiene. This result can be contrasted with the BMC/PMPS mixtures, in which the mixing volume change was negative. ${ }^{2}$ The consequences of a negative excess volume are contrary to the observed speeding up of the BMC relaxation and hence could not be the operative mechanism therein. ${ }^{2}$ Evidently in the present case, the decrease in density governs the effect the polymer has on the PCB dysamics, subsuming any contribution from the local friction or the relative $\tau_{0}$ 's. In general, both effects can be expected to modify the local relaxation processes in mixtures. In particular, we anticipate that whenever the higher $T_{\mathrm{g}}$ component of a mixture is more strongly coupled (in the respective neat states), its effect on the relaxation of the lower $T_{\mathrm{g}}$ component will be surprisingly small, or even qualitatively opposite from expectations based on conventional ideas about $T_{\mathrm{g}}$ and free volume. Chemical interaction between the components in a blend can, of course, also exert an influence on the glass transition temperatures ${ }^{20,33}$ and relaxation dynamics ${ }^{34}$ of mixtures.

Acknowledgment. This work was supported by the Office of Naval Research (in part by ONR Contract N0001494WX23010 (to K.L.N.)). P.G.S. expresses his gratitude for a National Research Council-Naval Research Laboratory postdoctoral fellowship.

\section{References and Notes}

(1) Lohfink, M.; Sillescu, H. In Proceedings of the 1st Tohwo University International Symposium; Kawasaki, K., Kawakatsu, T., Tokuyama, M., Eds.); American Institute of Physics: New York, 1992.

(2) Santangelo, P. G.; Roland, C. M.; Ngai, K. L.; Meier, G. Macromolecules 1993, 26, 6164.

(3) Lodge, T. P. J. Phys. Chem. 1993, 97, 1480.

(4) Schrag, J. L.; et al. J. Non-Cryst. Solids 1991, 131-133, 537.

(5) Ngai, K. L. J. Polym. Sci., Part B 1991, 29, 867.

(6) Gisser, D. J.; Ediger, M. D. Macromolecules 1992, 25, 1284.

(7) Fujita, H. Macromolecules 1993, 26, 643.

(8) Rizos, A.; Ngai, K. L. Phys. Rev. B 1992, 46, 8126.

(9) Ngai, K. L.; Rendell, R. W.; Rajagopal, A. K.; Teitler, S. Ann. N.Y. Acad. Sci. 1986, 484, 150.

(10) Ngai, K. L.; Rendell, R.W.J. Non-Cryst. Solids 1991, 131-133, 942 .

(11) Colmenero, J.; Arbe, A.; Alegria, A. Phys. Rev. Lett. 1993, 71, 2603.

(12) Roland, C. M.; Ngai, K. L. Macromolecules 1991, 24, 5315; 1992, $25,1844$.

(13) Colmenero, J.; Alegria, A.; Santangelo, P.G.; Ngai, K. L.; Roland, C. M. Macromolecules 1994, 27, 407.

(14) Minnick, M. G.; Schrag, J. L. Macromolecules 1980, 13, 1690.

(15) Morris, R. L.; Amelar, S.; Lodge, J. L. J. Chem. Phys. 1988, 89, 6523.

(16) von Meerwall, E. D.; Amelar, S.; Smeltzly, M. A.; Lodge, T. P. Macromolecules 1989, 22, 295.

(17) Patterson, D. Polym. Eng. Sci. 1982, 22, 64; Macromolecules $1969,2,672$.

(18) Lewis, G. N.; Randal, M.; Pitzer, K. S.; Brewer, L. Thermodynamics; McGraw-Hill: New York, 1961; Chapter 21.

(19) Maeda, Y.; Paul, D. R. J. Polym Sci., Polym. Phys. Ed. 1987, $25,1005$.

(20) Wolf, M.; Wendorff, J. H. Polymer 1989, 30, 1524.

(21) Ferry, J. D. Viscoelastic Properties of Polymers; Wiley: New York, 1980.

(22) Fujita, H. Fortschr. Hochpolym.-Forsch. 1961, 3, 1.

(23) Kohlrausch, R. Pogg. Ann. Phys. 1847, 12, 393.

(24) Williams, G.; Watts, D. C. Trans Faraday Soc. 1970, 66, 80.

(25) Adler, R. S.; et al. Macromolecules 1978, 11, 1058.

(26) Liu, Y.; Roy, A. K.; Jones, A. A.; Inglefield, P. T.; Ogden, P. Macromolecules 1990, 23, 968 .

(27) Adachi, K.; Hattori, M.; Ishida, Y. J. Polym. Sci., Polym. Phys. Ed. 1977, 15, 693

(28) Kambour, R. P.; Carbeck, J. D.; Nachlis, W. L. J. Non-Cryst. Solids 1991, 131-133, 563.

(29) Plazek, D. J.; Seoul, C.; Bero, C. A. J. Non-Cryst. Solids 1991 131-133, 570 .

(30) Roland, C. M.; Ngai, K. L. Macromolecules 1992, 25, 5765.

(31) Roland, C. M.; Ngai, K. L. Macromolecules 1993, 26, 6824.

(32) Flory, P. J. Selected Works of Paul J. Flory; Mandelkern, L.; Mark, J. E., Suter, U. W., Yoon, D. Y., Eds.; Stanford Press: Stanford, 1985; Vol. I.

(33) See, for example: Kwei, T. K.; Pearce, E. M.; Pennacchia, J. R.; Charton, M. Macromolecules 1987, 20, 174

(34) Roland, C. M.; Santangelo, P. G.; Baram, Z.; Runt, J. P. Macromolecules, submitted. 